\title{
Influence of an Sb doping layer in CIGS thin film solar cells: a photoluminescence study
}

\section{Van Puyvelde', J Lauwaert ${ }^{1}$, F Pianezzi ${ }^{2}$, S Nishiwaki ${ }^{2}$, P F Smet ${ }^{1}$, D Poelman ${ }^{1}$, A N Tiwari ${ }^{2}$ and H. Vrielinck $^{1}$}

${ }^{1}$ Department of Solid State Sciences, Ghent University, Krijgslaan 281-S1, 9000 Gent, Belgium

${ }^{2}$ Laboratory for Thin Films and Photovoltaics, Swiss Federal Laboratories for Materials Science and Technology (Empa), Ueberlandstrasse 129, CH-8600 Duebendorf, Switzerland

E-mail: Lisanne.VanPuyvelde@UGent.be

$\mathrm{Sb}$ doping of $\mathrm{Cu}(\mathrm{In}, \mathrm{Ga}) \mathrm{Se}_{2}$ (CIGS) solar cells has been reported to exhibit a positive effect on the morphology of the absorber layer, offering a possibility to lower manufacturing cost by lowering the annealing temperatures during the CIGS deposition. In this work electron microscopy, energy-dispersive X-ray spectroscopy and photoluminescence experiments have been performed on cells deposited on soda lime glass substrates, adding a thin Sb layer onto the Mo back contact prior to the CIGS absorber deposition. The defect structure of CIGS solar cells doped with Sb in this way has been investigated and is compared with that of undoped reference cells. The influence of substrate temperature during absorber growth has also been evaluated. For all samples the photoluminescence results can be explained by considering three donor-acceptor pair recombination processes involving the same defect pairs.

\section{Introduction}

Among the thin film solar cells, those based on a chalcopyrite $\mathrm{Cu}(\mathrm{In}, \mathrm{Ga}) \mathrm{Se}_{2}$ (CIGS) absorber layer are the most efficient, due to their direct band gap and favorable light absorption properties. One way to reduce the manufacturing cost is to lower the substrate temperature during absorber deposition. This temperature lowering also opens the opportunities to use light weight, flexible substrates (e.g. polymer substrates, metal foils). This broadens the range of applications and lowers the cost even further (cheaper substrates and roll-to-roll manufacturing). The lower temperature reduces problems as thermal stress and impurity in-diffusion.

It has been reported $[1,2]$ that $\mathrm{Sb}$ doping of the absorber promotes growth of the desired CIGS film grain structures under relatively mild thermal treatment conditions. Indeed, the larger grain sizes reduce recombination rates in the CIGS layer as less grain boundaries are present. Other studies $[3,4]$ did not report such unambiguously positive effect of Sb doping, however, implying it may have a more complex influence on the CIGS properties. In this study we investigate the effect of an Sb layer on the electrically active defects in the CIGS absorber by means of photoluminescence (PL) spectroscopy, while absorber morphology and composition are investigated with scanning electron microscopy (SEM) and energy dispersive X-ray analysis (EDX), respectively.

\section{Experimental}

A detailed description of the preparation can be found in [3]. Sb was incorporated in the solar cell structure by evaporating a $\mathrm{Sb}$ layer of $10 \mathrm{~nm}$ on top of a Mo coated soda-lime glass substrate, as shown in figure 1 .

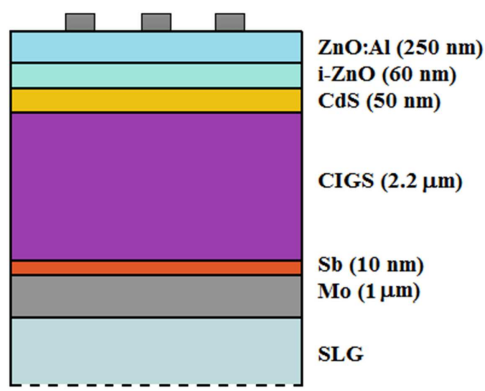

Figure 1. Structure of Sb-doped CIGS solar cell.

We label cells with such a $\mathrm{Sb}$ layer as $\mathrm{Sb}$ doped (SbD) and reference cells without Sb layer as non-doped (ND). The absorber is deposited by a three stage evaporation process. In order to prevent immediate evaporation of the Sb layer at the start of the first stage, the substrate temperature was initially kept low $\left(200{ }^{\circ} \mathrm{C}\right.$, see table 1$)$ and gradually 
increased during the growth $\left(400{ }^{\circ} \mathrm{C}\right.$ or $380{ }^{\circ} \mathrm{C}$, see table 1). For comparison the ND cells were also grown in this way. The maximum substrate temperature of cells made at the lowest temperature (LT) and higher temperature (HT), is below the 'standard' substrate temperature $\left(450^{\circ} \mathrm{C}\right)$. For completeness a reference cell grown at higher material substrate temperature $\left(460^{\circ} \mathrm{C}\right)$ was also investigated (HHT).

External quantum efficiency measurements (EQE) were carried out at room temperature in the $365 \mathrm{~nm}-1190 \mathrm{~nm}$ wavelength range, as described in [3].

Photoluminescence (PL) measurements were performed with a fluorescence spectrometer (FS920, Edinburgh Instruments). The PL was excited with a Xe arc lamp with double excitation monochromator and after monochromation detected with a liquid nitrogen-cooled germanium detector. In order to eliminate higher order diffraction signals from the excitation light, the emission was filtered with a high-pass filter $(670 \mathrm{~nm})$. For Scanning Electron Microscopy measurements (SEM) a FEI Quanta 200F FEG-SEM was used. For Electron Dispersive X-Ray analysis (EDX) a FEI Quanta 200 instrument, equipped with an EDAX Genesys 4000 setup was used. The acceleration voltage was $15 \mathrm{kV}$. The characteristic X-rays of $\mathrm{Cu}_{\mathrm{K}}, \mathrm{Se}_{\mathrm{L}}, \mathrm{Cd}_{\mathrm{L}}, \mathrm{Sb}_{\mathrm{L}}, \mathrm{In}_{\mathrm{L}}$ and $\mathrm{Ga}_{\mathrm{L}}$ were monitored.

\section{Results and discussion}

\subsection{Device and material characterization}

In table 1 the synthesis conditions of the cells taken up in this study are summarized, along with the results of solar cell performance measurements: open-circuit voltage $\left(\mathrm{V}_{\mathrm{OC}}\right)$, short-circuit current density $\left(\mathrm{J}_{\mathrm{SC}}\right)$, fill-factor $(\mathrm{FF})$ and efficiency $(\eta)$ under standard illumination conditions. One observes a slight decrease of the cell efficiency upon Sbdoping. The best cells are clearly those grown at the highest substrate temperature.

Table 1. Substrate temperature, device performance and band gap of the investigated cells.

\begin{tabular}{|c|c|c|c|c|c|c|c|c|}
\hline \multirow[b]{2}{*}{ Cell } & \multicolumn{2}{|c|}{$\begin{array}{l}\text { Tsubstrate } \\
\left({ }^{\circ} \mathrm{C}\right)\end{array}$} & \multirow[b]{2}{*}{$\mathrm{ND} / \mathrm{SbD}$} & \multirow[b]{2}{*}{$\begin{array}{l}\mathrm{V}_{\mathrm{oc}} \\
(\mathrm{mV})\end{array}$} & \multirow[b]{2}{*}{$\begin{array}{c}\mathbf{J}_{\mathrm{sc}} \\
\left(\mathrm{mA} / \mathrm{cm}^{2}\right)\end{array}$} & \multirow[b]{2}{*}{$\begin{array}{l}\mathrm{FF} \\
(\%)\end{array}$} & \multirow[b]{2}{*}{$\begin{array}{c}\eta \\
(\%)\end{array}$} & \multirow[b]{2}{*}{$\begin{array}{c}\mathrm{Eg} \\
(\mathrm{eV})\end{array}$} \\
\hline & $\begin{array}{c}1^{\text {st }} \\
\text { stage }\end{array}$ & $\begin{array}{l}2^{\text {nd }} / 3^{\text {rd }} \\
\text { stage }\end{array}$ & & & & & & \\
\hline HHT & $\begin{array}{l}200 \\
400\end{array}$ & 460 & ND & 716 & 31.6 & 74.1 & 16.8 & 1.161 \\
\hline \multirow[t]{2}{*}{ HT } & \multirow{2}{*}{$\begin{array}{l}200 \\
400\end{array}$} & \multirow{2}{*}{420} & ND & 681 & 31.5 & 72.4 & 15.5 & 1.121 \\
\hline & & & $\mathrm{SbD}$ & 648 & 30.0 & 73.2 & 14.3 & 1.129 \\
\hline \multirow{2}{*}{$\mathrm{LT}$} & \multirow{2}{*}{$\begin{array}{l}200 \\
380\end{array}$} & \multirow{2}{*}{380} & ND & 662 & 32.7 & 69.7 & 15.1 & 1.120 \\
\hline & & & $\mathrm{SbD}$ & 639 & 32.9 & 63.2 & 13.3 & 1.125 \\
\hline
\end{tabular}

Results of EQE measurements are shown in figure 2. To estimate the band gap $\left(E_{g}\right)$, the cut-off wavelength is determined as the intercept of the linear part of the long wavelength flank of the spectra with the EQE $=0$ axis. This cut-off wavelength underestimates $\mathrm{E}_{\mathrm{g}}$. The results can be found in the last column of table 1 . The $\mathrm{E}_{\mathrm{g}}$ values for LT and HT cells are similar; for the HHT cell it is markedly higher. There is, however, no clear distinction between the band gaps of $\mathrm{SbD}$ and ND cells. 


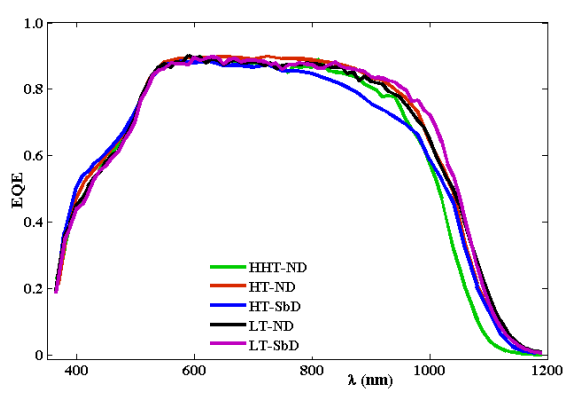

Figure 2. External Quantum Efficiency spectra of the devices.

SEM cross section images, shown in figure 3, were taken to investigate differences in film morphology, more specifically in grain size of the absorbers between cells. It is generally accepted that, in order to minimize recombination effects, grain sizes have to be large. Previous investigations [1, 2, 5] have reported a grain size increase upon Sb doping. In [4] the effect of Sb doping in single crystals is unclear. In agreement with the latter study, the SEM cross sections of the investigated cells reveal no clear differences in grain size between SbD and ND cells. On the other hand, an increase in grain size is observed when increasing the substrate temperature, in accordance with previous studies [1,5]. CIGS thin films grown at high substrate temperature have also been reported to exhibit a higher density [6], which may affect the interdiffusion between absorber and buffer layer, as will be discussed further on.
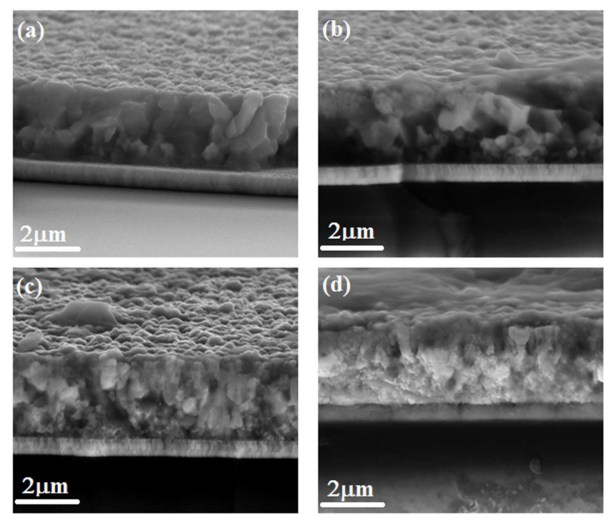

Figure 3. SEM cross section images of (a) HHT-ND, (b) HT-ND, (c) LT-ND, (d) LT-SbD.

As both substrate temperature [7] and Sb doping [3] may have an effect on the interdiffusion of the elements during the three stage evaporation, EDX line scan measurements have been performed. The resolution of the EDX scan is rather low, because of the large interacting volume, and a partial overlap of peak energies of certain monitored elements, which render the interpretation of the scan data difficult. Nonetheless the results of EDX line scans of crosssections of the cells are in accordance with composition measurements, reported in [3]. The absorber composition is $\mathrm{Cu}$ poor and exhibits a double graded Ga profile, with higher $\mathrm{Ga}$ content to the front and back of the film (figure 4). Such a dip in the Ga profile was recently reported as crucial for achieving high efficiency [8]. In addition, the EDX measurements in figure 4 are indicative of a larger in-diffusion of Cd into the CIGS absorber for the LT cells compared to the HT cells. As the chemical bath deposition of CdS was done in the same way for both samples, the thickness is expected to be the same. In literature, CIGS films grown at low substrate temperature have been reported to have a more $\mathrm{Cu}$ poor surface [7], where $\mathrm{Cu}$ vacancies can be occupied by $\mathrm{Cd}$. A higher in-diffusion in the LT cells may also result from a lower film density obtained at low substrate temperature [6]. For none of the samples an $\mathrm{Sb}_{\mathrm{L}}$ signal could be detected above the noise level.
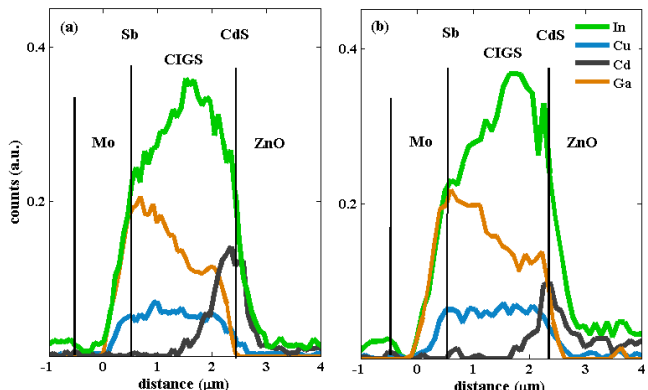

Figure 4. EDX line scan of cross section of In, $\mathrm{Cu}, \mathrm{Cd}$ and Ga profile (a) LT-ND, (b) HT-ND. 
In conclusion, based on the material characterization, no clear distinction can be made between SbD and ND cells. The device characterization, on the other hand, showed a small decrease in the cell efficiency upon Sb-doping. The positive effect of higher substrate temperature was observed both in the material and in the device characterization. In the following section, the defect composition of the cells is investigated using PL.

\subsection{Photoluminescence}

3.2.1 Excitation wavelength dependence. As we are primarily interested in defects in the absorber layer, we detected the PL of the samples in the near infrared range $\left(\mathrm{E} \leq \mathrm{E}_{\mathrm{g}}(\mathrm{CIGS}) \approx 1.15 \mathrm{eV}\right)$. PL emission scans are shown in figure 5, in which a marked influence of the excitation wavelength in the $300-650 \mathrm{~nm}$ range is observed.
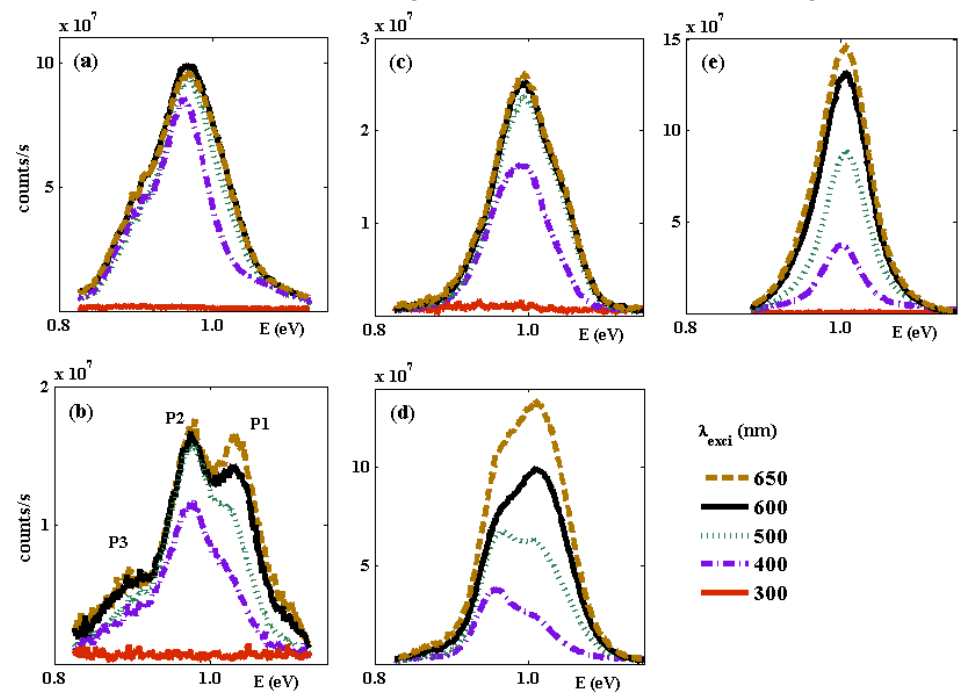

Figure 5. Photoluminescence emission at $5 \mathrm{~K}$ as a function of excitation wavelength. (a) LT-ND, (b) LT-SbD, (c) HTND, (d) HT-SbD (spectrum at $300 \mathrm{~nm}$ excitation not measured), (e) HHT-ND. Peak P1, P2 and P3 are indicated in (b).

$300 \mathrm{~nm}$ light does not excite PL from the absorber, as it is completely absorbed by the window layer ( $\mathrm{E}_{\mathrm{g}}(\mathrm{ZnO}: \mathrm{Al})$ $\sim 3.5 \mathrm{eV}(354 \mathrm{~nm})$ ). Light in the $\lambda=400-500 \mathrm{~nm}$ range is in part absorbed by the thin CdS buffer layer, but also penetrates in the absorber, resulting in a marked PL intensity. In this range the PL intensity exhibits an increase that can be readily explained by an incomplete absorption in the buffer layer. In the 500-650 nm excitation range, all light should be absorbed only in the CIGS layer. Nonetheless, (slight) changes in the PL spectral shape and intensity are still noted, in particular in the range of the highest energy peak for the SbD samples (figure $5 \mathrm{~b}$, d). This may be an indication of gradients in the defect distribution near the CdS/CIGS interface, related to the penetration depth of the excitation light in the absorber. Simulations [9] assuming a homogeneous CIGS layer show that the 1/e penetration depth in the absorber changes from 70 to $120 \mathrm{~nm}$ in the 400-650 nm wavelength range. The penetration depth may be further influenced by the specific Ga grading in the absorber.

In spite of the differences in shape among the different samples, all PL spectra can be decomposed in three broad Gaussian components, labeled as P1, P2 and P3 (figure 5b and figure 6). In view of the large band widths and overlap, the fitting error on these positions is estimated in the order of $5 \mathrm{meV}$. The energy of the peak maximum (at $10 \mathrm{~K}$ ) and difference in energy of the peak maximum can be found in table 2 .

Table 2. Peak positions and distances between them.

\begin{tabular}{lllllll}
\hline & \multicolumn{3}{c}{ Peak energy $(\mathrm{eV})$} & & \multicolumn{2}{c}{ Peak difference $(\mathrm{eV})$} \\
\cline { 2 - 4 } \cline { 6 - 7 } Cell & P3 & P2 & P1 & & P2-P3 & P1-P2 \\
\hline HHT & 0.945 & 1.005 & 1.059 & & 0.060 & 0.054 \\
HT-ND & 0.920 & 0.985 & 1.040 & & 0.065 & 0.055 \\
HT-Sb & 0.885 & 0.955 & 1.019 & & 0.070 & 0.064 \\
LT-ND & 0.897 & 0.967 & 1.036 & & 0.070 & 0.069 \\
LT-Sb & 0.910 & 0.976 & 1.037 & & 0.066 & 0.061 \\
\hline & & & & & \multicolumn{2}{c}{ Average $(\mathrm{eV})$} \\
\cline { 6 - 7 } & & & & 0.066 & 0.061 \\
\hline
\end{tabular}


The PL of the HHT cell (figure 5e) is shifted towards higher energy as a result of the higher band gap: the difference in cut-off energy via EQE measurements at RT is $37 \mathrm{meV}$, the difference in maximum peak energy of peak $\mathrm{P} 2$ is about $34 \mathrm{meV}$ at $5 \mathrm{~K}$. The distance between the $\mathrm{P} 1$ and $\mathrm{P} 2$ positions and between $\mathrm{P} 2$ and $\mathrm{P} 3$ is similar for all cells. This suggests that same defects are involved in the different cells. The peak at highest energy (P1) is most pronounced for the SbD cells (figure 6a). The second peak (P2) is clearly visible for all samples and is the dominant peak in the ND cells. The lowest energy peak (P3) is most clearly distinguished for the LT cells (figure 6b).
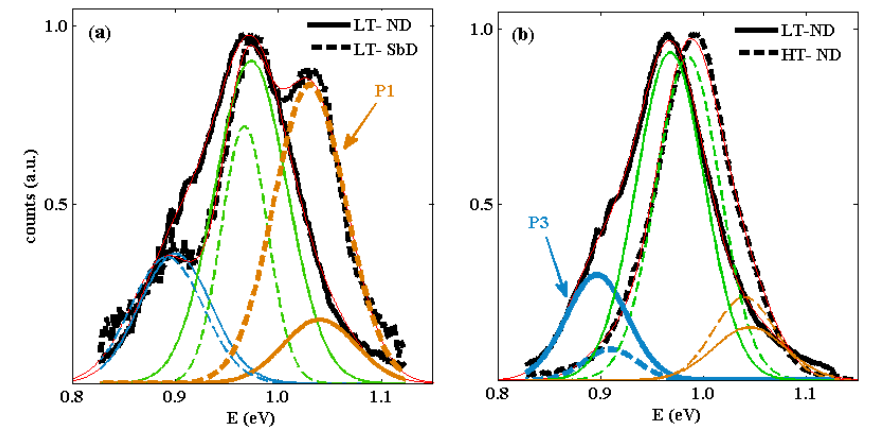

Figure 6. Comparison of normalized PL spectra for (a) LT-ND and LT-SbD cells $\left(\lambda_{\text {exci }}=650 \mathrm{~nm}, \mathrm{~T}=5 \mathrm{~K}\right.$ ); (b) LT-ND and HT-ND cells $\left(\lambda_{\text {exci }}=500 \mathrm{~nm}, \mathrm{~T}=10 \mathrm{~K}\right)$.

All peaks are quite broad and the spectra do not exhibit phonon replicas nor exciton transitions. This is a clear signature of highly compensated CIGS material [10]. As concluded from the compositional study, the CIGS absorber is $\mathrm{Cu}$-poor. Hence it is expected to exhibit a high concentration of copper vacancies, providing the p-type conductivity of the material. It has been shown previously that Cu-poor CIGS is highly compensated [11, 12], having comparable and high density of donors and acceptors [13]. Most of the defects are charged without generating free carriers. The resulting local variation of the fixed space charge cannot be completely screened by the comparatively low free carrier density. This causes a distortion of the band structure. The average depth of potential fluctuations is dependent on the total charged impurity concentration and the screening by the free carriers.

Peaks in the PL spectra of a highly compensated semiconductor are usually assigned to donor-acceptor pair DAP recombination [11]. The tunneling of carriers between spatially separated donors and acceptors, at different local potential, can considerably reduce the transition energy. A broad range of energies is hence expected for the (DAP) transitions, leading to broad spectra. In the presence of band fluctuations the emission energy for DAP recombination is given by

$$
\mathrm{E}_{\text {DAP_comp }}=\mathrm{E}_{\mathrm{DAP}}-2 \gamma
$$

where $\gamma$ represents the average depth of the potential fluctuations. Hence, potential fluctuations hamper an accurate determination of the donor and acceptor binding energies from

$$
E_{D A P}=E_{g}-E_{D}-E_{A}
$$

applicable for a widely separated DAP, ignoring the Coulomb interaction between the donor and acceptor.

The theory developed for DAP recombination in a highly compensated semiconductor also predicts distinctive excitation power and temperature dependencies of the PL as a result of potential fluctuations $[11,13,14]$ which are examined in the following subsections.

3.2.2. Excitation power dependence. Excitation power dependent PL measurements at low temperature $(5 \mathrm{~K})$ have been performed. The excitation power $\left(\mathrm{P}_{\text {exci }}\right)$, measured in front of the lens located before the cryostat, was in the order of $100 \mathrm{~mW} / \mathrm{m}^{2}\left(\lambda_{\text {exci }}=500 \mathrm{~nm}\right)$. Gray filters were placed in front of the cryostat window in order to decrease the excitation power. In figure 7 the excitation power dependence of the PL maximum position is shown for those samples whose PL maximum corresponds to the P2 peak. The results for the HT-SbD cell are not shown: the analysis of the spectra is complicated by nearly equal contributions of P1 and P2. 


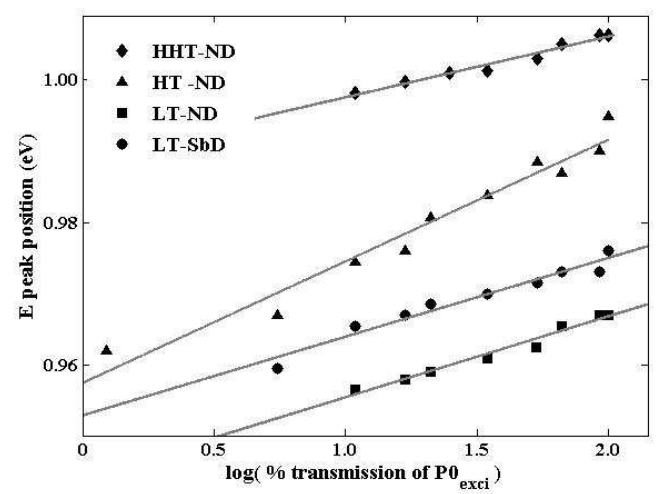

Figure 7. Emission peak energy as a function of excitation power. The solid lines represent fittings assuming a linear relation between the peak position $(\sim \mathrm{P} 2)$ and the logarithm of excitation power, yielding shifts of $8.5,17,11.4$ and

$11.1 \mathrm{meV} /$ decade for HHT-ND, HT-ND, LT-ND and LT-SbD cells, respectively.

With increasing excitation power, a blue-shift between 8.5 and $17 \mathrm{meV} / \mathrm{dec}$ ade is found. This large blue- shift is a second indication of potential fluctuations in PL measurements [13]. Indeed, with increasing excitation power, an increasing number of defects are neutralized by carrier trapping and the density of free charge carriers increases, screening the remaining charged defects more effectively. This results in a flattening of the fluctuating potentials, leading to a blue-shift in the peak energy position.

In material without potential fluctuations, a small blue-shift with increasing excitation power is also expected for DAP luminescence. This is a result of the decrease in the average distance between the donor and acceptor involved in the recombination as excitation power increases. However, for CIGS a maximal shift of 1-2 meV/decade excitation power is expected for this effect [11].

3.2.3. Temperature dependence. Photoluminescence spectra as a function of temperature reveal a pronounced red-shift of the spectra upon increasing temperature. Over the complete measured temperature range a decomposition in three Gaussian peaks allows a good reproduction of the spectra. In figure 8 the temperature dependence of the peak positions for the spectra at an excitation wavelength of $500 \mathrm{~nm}$ for the HT-ND and HT-SbD cell is shown. For the P2 peak of the HT-ND cell a red-shift of $0.9 \mathrm{meV} / \mathrm{K}$ was observed and for the P2 peak of the HT-SbD cell $0.8 \mathrm{meV} / \mathrm{K}$. The shift to lower energy with increasing temperature cannot be explained by the decrease of the band gap alone. Moreover, it has been reported that the band gap of chalcopyrites is essentially constant below $100 \mathrm{~K}$ [12]. The redshift is yet another indication of potential fluctuations in compensated material $[11,13]$. At low temperature no complete thermalization of the carriers over the different wells is possible and carriers occupy wells close to where they are created. When temperature increases, thermalization of the carriers into deeper wells becomes possible, resulting in a red-shift. For all cells, the PL intensity also decreases as temperature increases. In view of the presence of three overlapping peaks in all spectra, the temperature dependence of intensity is rather complicated and differs for different cells. Its analysis is not attempted here.

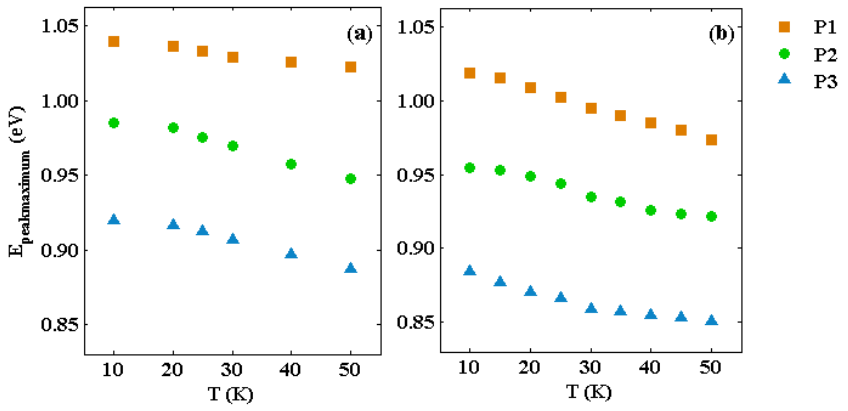

Figure 8. Temperature dependence of the three peak positions for (a) HT-ND and (b) HT-SbD ( $\left.\lambda_{\text {exci }}=500 \mathrm{~nm}\right)$.

\subsection{Defect identification}

As mentioned before, the PL signals originate from the CIGS layer close to the interface with the buffer, relatively far from the back contact and the Sb layer. The PL experiments reveal a marked influence of both the substrate temperature during the absorber growth and of Sb doping on the defect distribution in CIGS near the buffer/absorber interface. Moreover, the spectral width, the excitation power and temperature dependence of the PL signal are compatible with DAP recombination in a semiconductor with strong potential fluctuations. The lack of direct information about the magnitude of the potential fluctuations hampers the calculation of the defect binding energies 
$\mathrm{E}_{\mathrm{D}}+\mathrm{E}_{\mathrm{A}}$. Moreover, the $\mathrm{EQE}$ measurements at $\mathrm{RT}$ only yield an underestimate of the band gap values at low temperatures, further complicating the determination of $\mathrm{E}_{\mathrm{D}}+\mathrm{E}_{\mathrm{A}}$. In addition, reported defect identifications in CIGS, based on binding energies for donors and acceptors, are often conflicting. In the following, an identification is proposed based on literature results, where we mainly make use of the distances between the PL peaks as given in table 2, and on the stoichiometry of the samples as evidenced by SIMS [3] and EDX results. Other interpretations may, however, still be possible.

Since the material is $\mathrm{Cu}$-poor, we assume that the acceptor of the DAP is the copper vacancy $\left(\mathrm{V}_{\mathrm{Cu}}\right)$. Based further on the stoichiometry of the samples and theoretical predictions of the formation energy of defects [15], $\mathrm{V}_{\mathrm{Se}}$ and $\mathrm{In}_{\mathrm{Cu}}$ are expected to be the most abundant intrinsic donors in CIGS, compensating the $\mathrm{V}_{\mathrm{Cu}}$ acceptors. In [16] PL experiments (as a function of temperature and excitation power) on co-evaporated $\mathrm{CuInSe} \mathrm{e}_{2}$ thin films with varying $\mathrm{Cu} / \mathrm{In}$ ratio were performed. The emission spectrum of $\mathrm{Cu}$-poor material showed two broad emission peaks which were assigned to $\mathrm{V}_{\mathrm{Cu}}-\mathrm{V}_{\mathrm{Se}}$ and $\mathrm{V}_{\mathrm{Cu}}-\mathrm{In}_{\mathrm{Cu}}$ DAP recombination.

Adopting also this identification for P1 and P2, the PL results indicate that $\operatorname{In}_{\mathrm{Cu}}$ (associated with P2) is the dominant donor defect in most of the cells, especially in the ND, at least in the region close (100-200 $\mathrm{nm}$ ) to the buffer/absorber interface. The results further indicate that $\mathrm{Sb}$-doping leads to a higher concentration in the $\mathrm{V}_{\mathrm{Se}}$ donor defect (associated with P1), which in addition appears to exhibit a positive gradient towards the absorber bulk. This effect of Sb doping could be related to a hindered diffusion of $\mathrm{Na}$ in the absorber layer due to the Sb layer. As shown in $[7,17,18]$ the presence of $\mathrm{Na}$ in the CIGS film and/or surface suppresses Se diffusion via grain boundaries and in this way impedes Se loss. In [2] it was reported that Na diffusion into the absorber layer is hindered by the presence of an Sb layer on top of the Mo contact. A lower Na concentration in Sb doped cells might thus lead to a higher Se loss and hence a higher $\mathrm{V}_{\mathrm{Se}}$ concentration.

Finally, the PL measurements reveal an increased contribution of peak P3 in LT cells. In $[19,20]$ it has been shown that chemical bath deposition of CdS on CIGS may lead to changes in the PL spectrum, introducing a low energy peak. The associated deep electronic level, at $\mathrm{E}_{\mathrm{C}}-210 \mathrm{meV}$ [19] was assigned to the $\mathrm{Cd}_{\mathrm{Cu}}$ donor, formed by the substitution of $\mathrm{Cu}$ by $\mathrm{Cd}$ in the $\mathrm{Cu}$-poor material. This substitution is considered very likely because the ionic radii of $\mathrm{Cd}^{2+}(0.097 \mathrm{~nm})$ and $\mathrm{Cu}^{+}(0.096 \mathrm{~nm})$ are very similar [21]. It was furthermore suggested that a higher $\mathrm{Cu}$ deficiency near the buffer/absorber surface enhances the $\mathrm{Cd}_{\mathrm{Cu}}$ concentration. In [22] Cd diffusion along the grain boundaries was found. In accordance with these studies, the low energy peak P3 may be identified as $\mathrm{Cd}_{\mathrm{Cu}}-\mathrm{V}_{\mathrm{Cu}} \mathrm{DAP}$ recombination.

Our PL results do not yield direct evidence for Sb incorporation in the absorber. Sb might substitute Se, which would introduce extra acceptors in CIGS. Electrical spectroscopy experiments might give further insight concerning this issue. $\mathrm{C}-\mathrm{V}$ measurements do not reveal a clear difference between undoped and Sb-doped cells. Electrical spectroscopy experiments, e.g. Admittance Spectroscopy, Deep-Level Transient Spectroscopy or Deep-Level Capacitance Profiling may give further insight concerning this issue. Such measurements are, however, beyond the scope of the present article.

\section{Conclusion}

The influence of a thin Sb layer evaporated on the Mo back contact in CIGS cells processed by multistage evaporation at reduced substrate temperatures was investigated. As shown in a previous study on these cells [3], Sb incorporation does not lead to improvement of the efficiency of these cells. EQE and SEM measurements showed a clear effect of substrate temperature, but no obvious effect of the Sb layer. The PL spectra, on the other hand, do indicate effects of both substrate temperature and Sb doping on the defect composition in the CIGS absorber. For all the cells the PL spectra exhibit three broadened Gaussian peaks, which red-shift with increasing temperature and blue-shift with increasing excitation power. These are features of potential fluctuations in $\mathrm{Cu}$-poor highly compensated CIGS. For low temperature cells a PL peak at low energy attributed to DAP recombination between $\mathrm{V}_{\mathrm{Cu}}$ and $\mathrm{Cd}$ in-diffusion related donors is more pronounced. Sb doped cells have a more pronounced peak at high energy. The PL results indicate a positive concentration gradient in the associated donor defect, probably $\mathrm{V}_{\mathrm{Se}}$, towards the absorber bulk.

\section{References}

[1] Yuan M, Mitzi D B, Gunawan O, Kellock A J, Chey S J and Delin V R 2010 Antimony assisted low-temperature processing of $\mathrm{CuIn}_{1-\mathrm{x}} \mathrm{Ga}_{\mathrm{x}} \mathrm{Se}_{2-\mathrm{y}} \mathrm{S}_{\mathrm{y}}$ solar cells Thin Solid Films 519 852-6

[2] Nakada T, Honishi Y, Yatsushiro Y and Nakakoba H 2011 Impacts on Sb and Bi incorporations on CIGS thin films and solar cells Photovoltaic Specialists Conf. (Seattle)

[3] Nishiwaki S, Pianezzi F, Fella C M, Kranz L, Bücheler S and Tiwari A N 2012 Reduction of CIGS deposition temperature by addition of an Sb precursor Conf. Proc. EUPVSEC (Frankfurt) 
[4] Ben Rabeh M, Chaglabou N and Kanzari M 2010 Effect of antimony incorporation on structural properties of $\mathrm{CuInS}_{2}$ crystals Physics Research B 268 273-6

[5] Zhang S, Wu L, Yue R, Yan Z, Zhan H and Xiang Y 2013 Effects of Sb-doping on the grain growth of Cu(In,

Ga)Se ${ }_{2}$ thin films fabricated by means of single-target sputtering Thin Solid Films 527 137-40

[6] Zhang L, He Q, Jiang W, Liu F, Li C and Sun Y 2009 Effects of substrate temperature on the structural and electrical properties of $\mathrm{Cu}$ (In, Ga)Se $\mathrm{S}_{2}$ thin films Sol. Energy Mater. Sol. Cells 93 114-8

[7] Rudmann D, Brémaud D, da Cunha A F, Bilger G, Strohm A, Kaelin M, Zogg H and Tiwari A N 2005 Sodium incorporation strategies for CIGS growth at different temperatures Thin Solid Films 480 55- 60

[8] Chirilă A et al. 2011 Highly efficient $\mathrm{Cu}(\mathrm{In}, \mathrm{Ga}) \mathrm{Se}_{2}$ solar cells grown on flexible polymer films Nature Mater 10 $857-61$

[9]Burgelman M, Nollet P and Degrave S 2000 Modelling polycrystalline semiconductor solar cells Thin Solid Films $361527-32$

[10] Levanyuk A P and Osipov V V 1981 Edge luminescence of direct-gap semiconductors Sov. Phys. Usp. 24 187-215

[11] Schumacher S A and Botha J R 2006 Photoluminescence study of potential fluctuations in thin layers of $\mathrm{Cu}\left(\mathrm{In}_{0.75} \mathrm{Ga}_{0.25}\right)\left(\mathrm{S}_{y} \mathrm{Se}_{1-\mathrm{y}}\right)_{2}$ J. Appl. Phys 99 1-8

[12] Siebentritt S, Papathanasiou N and Lux-Steiner M C 2006 Potential fluctuations in compensated chalcopyrites Physica B 376 831-3

[13] Siebetritt S and Rau U 2006 Wide-Gap Chalcopyrites vol 86 ed Hull R, Osgood $R M$ et al (New York: Springer) pp 113-48

[14] Yu P W 1977 Excitation-dependent emission in Mg-, Be -, Cd-, and Zn-implanted GaAs J. Appl. Phys 48 504351

[15]Zhang S B , Wei S and Zunger A 1998 Defect physics of the CuInSe ${ }_{2}$ chalcopyrite semiconductor Phys. Rev. B $579642-55$

[16] Zott S, Leo K, Ruckh M and Schock H 1996 Photoluminescence of polycrystalline CuInSe ${ }_{2}$ thin films Appl. Phys. Lett. 68 1144-46

[17] Rudmann D, da Cunha A F, Kaelin M, Kurdesau F, Zogg H, Tiwari A N and Bilger G 2004 Efficiency enhancement of $\mathrm{CuInGaSe}_{2}$ solar cells due to post-deposition Na incorporation Appl. Phys. Lett 84 1129-31

[18] Rudmann D, Brémaud D, Zogg A and Tiwari A N 2005 Na incorporation into CuInGaSe ${ }_{2}$ for high-efficiency flexible solar cells on polymer foils J. Appl. Phys 97 1-5

[19] Shirakata S and Nakada T 2009 Photoluminescence and time-resolved photoluminescence in $\mathrm{Cu}(\mathrm{In}, \mathrm{Ga}) \mathrm{Se}_{2}$ thin films and solar cells Phys. Status Solidi C 6 1059-62

[20] Ramanathan K, Noufi R, Granata J, Webb J and Keane J 1998 Prospects for in situ junction formation in CuInSe ${ }_{2}$ based solar cells Sol. Energy Mater. Sol. Cells 55 15-22

[21] Nakada T 2000 Nano-structural investigations on Cd-doping into $\mathrm{Cu}(\mathrm{In}, \mathrm{Ga}) \mathrm{Se}_{2}$ thin films by chemical bath deposition process Thin Solid Films $361346-52$

[22] Cojocaru-Mirédin O, Choi O, Wuerz R and Raabe D 2012 Exploring the p-n junction region in $\mathrm{Cu}(\mathrm{In}, \mathrm{Ga}) \mathrm{Se}_{2}$ thin-film solar cells at the nanometer-scale Appl. Phys. Lett 101 1-5 